EPJ Web of Conferences 113, 01003 (2016)

DOI: $10.1051 /$ epjconf/201611301003

(C) Owned by the authors, published by EDP Sciences, 2016

\title{
Cluster Symmetries and Dynamics
}

\author{
Martin Freer $^{1, a}$ \\ ${ }^{1}$ School of Physics and Astronomy, University of Birmingham, Birmingham, B15 2TT, UK
}

\begin{abstract}
Many light nuclei display behaviour that indicates that rather than behaving as an $A$-body systems, the protons and neutrons condense into clusters. The $\alpha$-particle is the most obvious example of such clustering. This contribution examines the role of such $\alpha$-clustering on the structure, symmetries and dynamics of the nuclei ${ }^{8} \mathrm{Be},{ }^{12} \mathrm{C}$ and ${ }^{16} \mathrm{O}$, recent experimental measurements and future perspectives.
\end{abstract}

\section{Introduction}

The behaviour of nuclei as a function of their internal energy is rich and varied. Collective degree of freedom result of transferal to rotational modes, above decay threshold particle emission is possible and if those decay thresholds are connected with the decay of composite particles, e.g. $\alpha$-particles, then cluster emission can occur. Particularly in light nuclei, the nature of the intrinsic structure prior to such cluster decays is found to be closely related to the decay partition. This has been encapsulated in the Ikeda diagram [1] (Fig. 1). Here as the internal energy of the nucleus is increased to the point an $\alpha$-decay threshold is encountered then the nucleus is able to dissipate the internal energy into the mass of the clusters. For example, at an excitation energy of $7.27 \mathrm{MeV}$ the $3 \alpha$ decay threshold in ${ }^{12} \mathrm{C}$ is encountered. The Ikeda picture would suggest that at this point that one possible structural mode for ${ }^{12} \mathrm{C}$ is that of $3 \alpha$-clusters. Interestingly, the $3 \alpha$-decay threshold is lower than in energy than the ${ }^{8} \mathrm{Be}+\alpha$ two-body decay threshold (Fig. 2). The ${ }^{8} \mathrm{Be}$ nucleus is, however, itself unbound to decay to $2 \alpha$-particles by $93 \mathrm{keV}$. So by the same arguments ${ }^{8} \mathrm{Be}$ would contain a $2 \alpha$-cluster structure.

Excited states located just above such decay thresholds thus have the maximum structural overlap with such clusters, with increasing excitation energy allowing additional degrees of freedom to mix. Famously, the $7.65 \mathrm{MeV}$ Hoyle state (Fig. 2) has been linked to $3 \alpha$-cluster structure (as outlined in Ref. [2] and references therein), which has been linked, in turn, to the triple $\alpha$-process [3, 4] responsible for the synthesis of carbon-12. Here, first two $\alpha$-particles fuse to form ${ }^{8} \mathrm{Be}$ whose $\alpha$-decay results in an equilibrium concentration of ${ }^{8} \mathrm{Be}$. The second step is the capture by ${ }^{8} \mathrm{Be}$ to form ${ }^{12} \mathrm{C}$ at an excitation above the ${ }^{8} \mathrm{Be}+\alpha$ threshold followed by electromagnetic decay to the ${ }^{12} \mathrm{C}$ ground state (see Fig. 2). Hoyle recognised the need for a $J^{\pi}=0^{+}$state close to this energy in order to account for the absolute abundance of ${ }^{12} \mathrm{C}$ and the relative abundance of ${ }^{12} \mathrm{C}$ and ${ }^{16} \mathrm{O}$ [5]. The presence of the Hoyle-state at $7.65 \mathrm{MeV}$ resonantly boosts the capture process by a factor of close to 10-100 million. Hoyle predicted the existence of a state at $7.68 \mathrm{MeV}$ [5], and whilst visiting Caltech, convinced the local group to search for the state. They measured the ${ }^{14} \mathrm{~N}(\mathrm{~d}, \alpha){ }^{12} \mathrm{C}$ reaction using a high resolution spectrometer at which point a state at $7.68 \mathrm{MeV}$ was observed [6]. Subsequent measurements refined

a e-mail: M.Freer@bham.ac.uk 


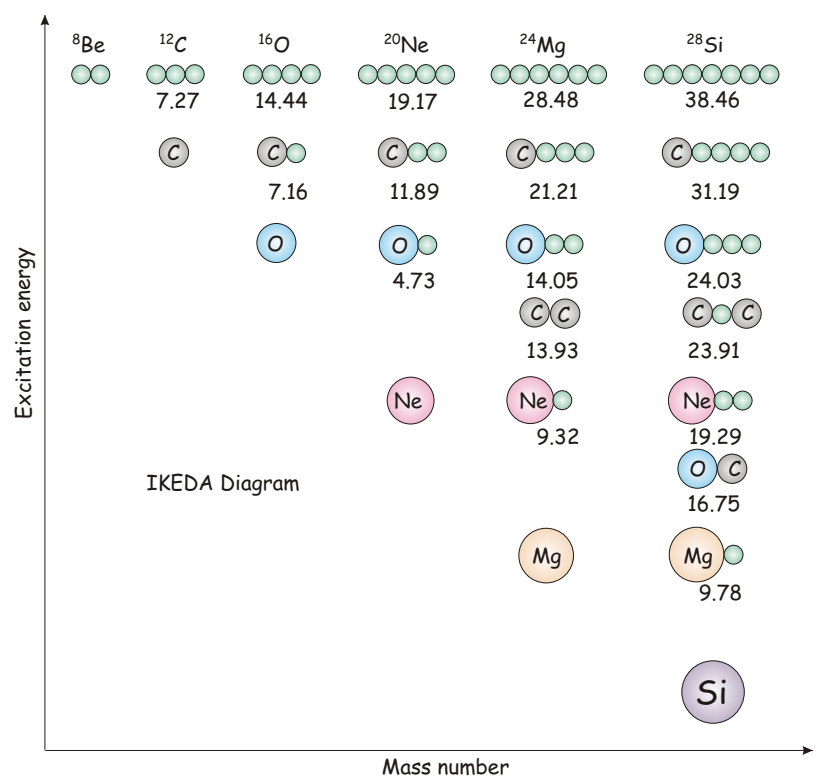

Figure 1. The Ikeda diagram [1]. This approach reveals that cluster structures, rather than appearing in the ground-state, should appear close to the cluster decay thresholds. At this point there is the possibility to convert the internal excitation energy into the mass of the cluster constituents.

the energy of the state to $7.653 \pm 0.008 \mathrm{MeV}$ and indicated the most probable spin and parity to be $0^{+}$[7]. The connection between the existence of organic life, and ultimately human-kind, has led to the interpretation [8] that the prediction of the existence of 7.65 MeV state by Hoyle was an example of the anthropic principle, an idea introduced by Carter in 1973 [9]. The principle relies on the fact that intelligent life exists, to assert certain properties of the universe must exist, i.e. we exist therefore so must the $7.65 \mathrm{MeV}$ state in ${ }^{12} \mathrm{C}$. The question as to if Hoyle deployed the anthropic principle or not has been the subject of some debate, reviewed by Kragh [10].

The existence of $0^{+}$cluster states close to the $\alpha$-decay thresholds of ${ }^{8} \mathrm{Be}$ and ${ }^{12} \mathrm{C}$ is mirrored in other light nuclei, which are $\alpha$-conjugate systems. In ${ }^{16} \mathrm{O}$ the $\alpha$-decay threshold is $7.16 \mathrm{MeV}$ and the first excited $0^{+}$state lies at $6.05 \mathrm{MeV}$. This latter state has been identified with a ${ }^{12} \mathrm{C}+\alpha$ cluster structure [11]. In this instance the Ikeda picture is fulfilled. It is interesting to ponder why this might be the case: why is it that nature arranges for such cluster states to be close to the decay thresholds, recognising the mass partition? Weakly bound nuclei close to decay thresholds can be thought of as open quantum systems where the properties of the unbound states influence, or couple to, the bound states below the threshold. Okołowicz, Nazarewicz and Płoszajczak have recently explored the link between the appearance of cluster states at threshold and the role of the continuum [12]. This is also discussed in Ref. [13]. 


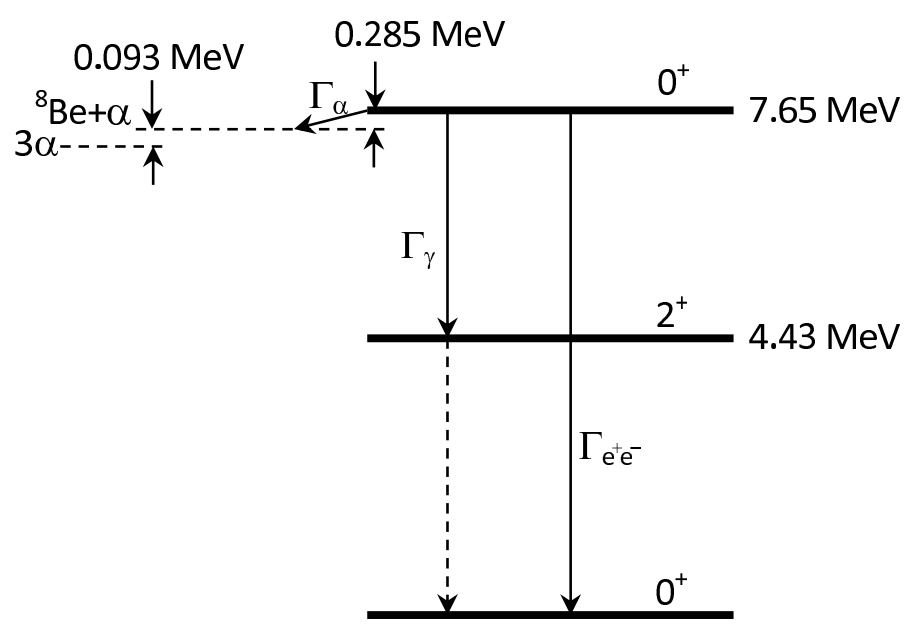

Figure 2. The energy levels of ${ }^{12} \mathrm{C}$ relevant for the triple- $\alpha$ process. The synthesis of ${ }^{12} \mathrm{C}$ takes place in two stages (i) the formation of ${ }^{8} \mathrm{Be}$ and then (ii) ${ }^{8} \mathrm{Be}+\alpha \rightarrow{ }^{12} \mathrm{C}$. The $7.65 \mathrm{MeV}$ Hoyle state promotes the reaction rate associated with this second step by close to eight orders of magnitude. The presence of the Hoyle-state close to the $3 \alpha$ decay threshold, would suggest it possesses a $3 \alpha$ cluster structure.

The transition from a shell-model ground-state, where the degrees of freedom are those of a strongly correlated single-particle system, to a clustered excited state, mirror the transitions that take place in the complex many-body system of water. Water is a many body system where the interplay between hydrogen bonds and van der Waals interactions not only describe the bulk fluid but the dynamics of the formation of water clusters $[14,15]$. Such clusters are challenging to observe from the experimental perspective. As heat is added to water, the excess energy results in the formation of steam or water vapour. Here these droplets can be viewed as liberated water clusters. In the nuclear analogue, as energy is added, $\alpha$-particles can be evaporated - when the decay threshold is reached. If clusters can exist in water, can they also form within the fermionic neutron-proton nuclear fluid?

Fig. 3 shows a modified version of the Ikeda diagram which shows the water-line linked with the explicit precipitation of clusters above, and the shell-model like structures below. The question as to the structure of nuclei above and below this line can at least be answered from the theoretical perspective. The two densities shown in Fig. 3 correspond to Antisymmetrized Molecular Dynamics (AMD) calculation for ${ }^{12} \mathrm{C}$ [16]. The densities show the resulting intrinsic structures for the groundstate, $0_{1}^{+}$, and second $0^{+}$state, $0_{2}^{+}$. Both reveal the three $\alpha$-structure, with it being more explicit in the case of the excited state, which is linked to the ${ }^{12} \mathrm{C}$ Hoyle-state. However, it is clear that the triangular symmetry is present also in the ground-state. The energy levels of ${ }^{12} \mathrm{C}$ calculated using the AMD approach are shown in Fig. 4. There is seen to be excellent agreement with experiment, a feature which is not found in the case of mean-field inspired models such as the no core shell model (NCSM) [19].

An experimental verification of the structure of ${ }^{12} \mathrm{C}$ linked to these two intrinsic configurations has been the subject of intensive experimental work. 


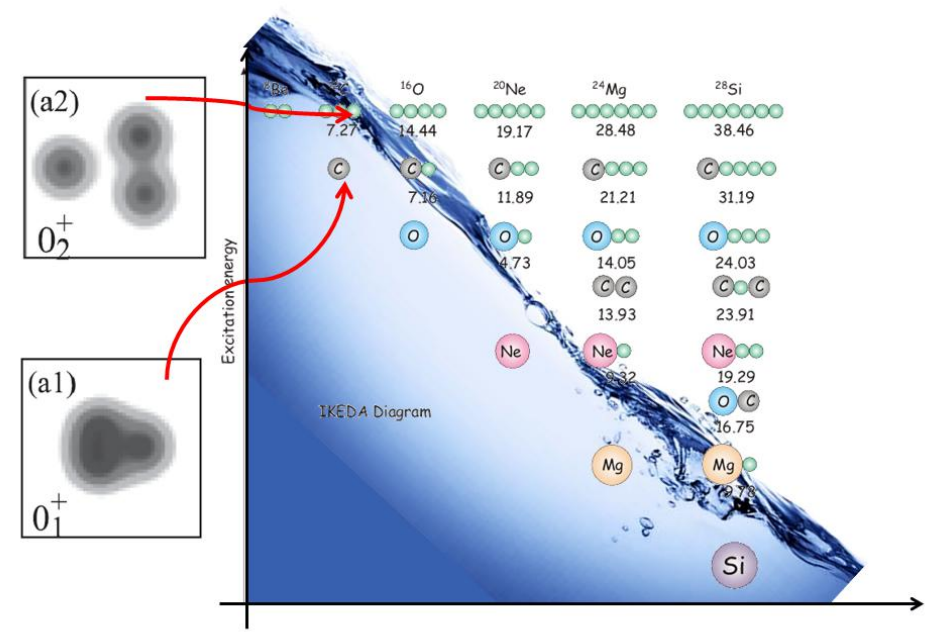

Figure 3. An adapted version of the Ikeda diagram, Fig. 1, delineating the change in structure that occurs above and below the $\alpha$-decay threshold. The AMD calculations [16] for ${ }^{12} \mathrm{C}$ show that above the threshold $\left(0_{2}^{+}\right)$the $3 \alpha$ cluster structure is evident. For the ground state, $0_{1}^{+}$, the cluster structure is still apparent, but the clusters are in a more compact configuration.

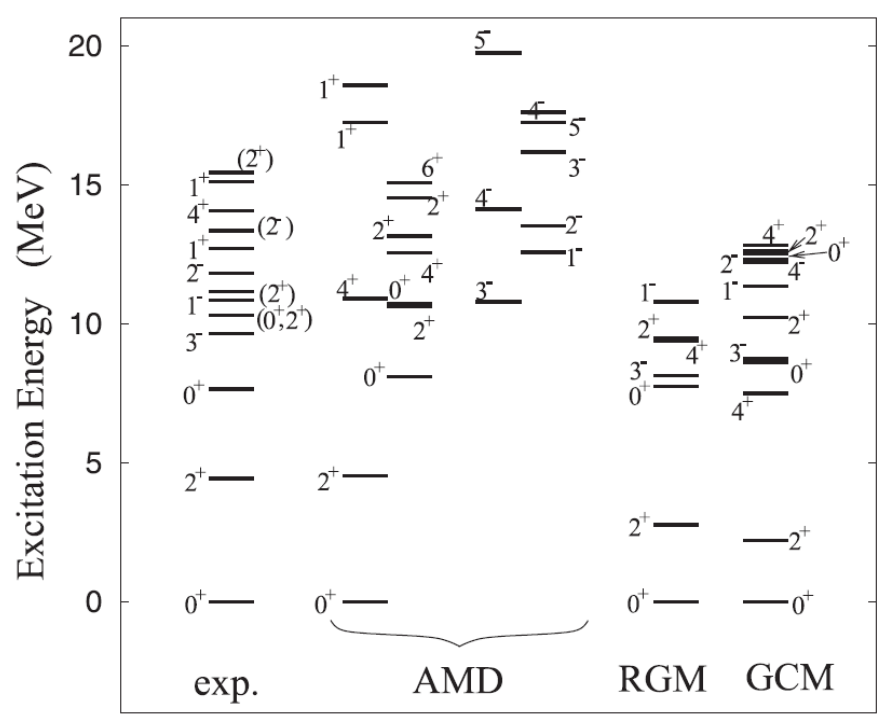

Figure 4. Energy levels for ${ }^{12} \mathrm{C}$ predicted by the AMD approach [16], resonanting group method (RGM) [17] and generator coordinate method (GCM) [18], from [16]. 


\section{Experimental Signatures for the $3 \alpha$ System}

In 1938 Hafstad and Teller [20] identified for $\alpha$-particle systems that there should be a set of dynamical symmetries. In the case of ${ }^{12} \mathrm{C}$ the dynamical symmetries of $3 \alpha$-system correspond to a spinning top with a triangular point symmetry $\left(D_{3 h}\right)$. The rotational properties of these states are given by

$$
E_{J, K}=\frac{\hbar^{2} J(J+1)}{2 I_{B e}}-\frac{\hbar^{2} K^{2}}{4 \mathcal{I}_{B e}}
$$

where $\mathcal{I}_{B e}$ is the moment of inertia corresponding to two touching $\alpha$-particles, which can be determined from the ${ }^{8} \mathrm{Be}$ ground-state rotational band [20]. $K$ is the projection of the angular momentum onto the symmetry axis of the $3 \alpha$ system.

One would expect that based on this structure there should be a number of rotational bands with different values of $K$. For $K^{\pi}=0^{+}$, the rotations will be around an axis which lies in the plane of the three $\alpha$-particles, generating a series of states $0^{+}, 2^{+}, 4^{+} \ldots$. These correspond to the rotation of a ${ }^{8} \mathrm{Be}$ nucleus - the rotation axis passing through the centre of the third $\alpha$-particle. The next set of rotations corresponds to the rotation around an axis perpendicular to the plane of the triangle, with each $\alpha$-particle having one unit on angular momentum - giving $L=3 \times 1 \hbar ; K^{\pi}=3^{-}$. Rotations around this axis and that parallel to the plane combine to give a series of states $3^{-}, 4^{-}, 5^{-} \ldots$. The next set of collective states then correspond to each $\alpha$-particle having $L=2 ; K^{\pi}=6^{+} \ldots$ Such an arrangement possesses a $D_{3 h}$ point group symmetry.

\subsection{Excited States Linked to the Hoyle-state}

If the structure of the excited Hoyle-state was close to that of the ground state, then the coupling of rotational modes would then produce a corresponding $2^{+}$state at $4.4 \mathrm{MeV}$ (the first excited state in ${ }^{12} \mathrm{C}$ is at $4.4 \mathrm{MeV}$ ) above $7.65 \mathrm{MeV}$, i.e. $12.05 \mathrm{MeV}$. There is no known $2^{+}$state at this energy, this itself points to a more complex structure. The closest state which has been reported with $J^{\pi}=2^{+}$is at $11.16 \mathrm{MeV}$ [21]. This state was originally observed in the ${ }^{11} \mathrm{~B}\left({ }^{3} \mathrm{He}, d\right){ }^{12} \mathrm{C}$ reaction, however has not been observed in measurements subsequently. A re-measurement of this reaction using the K600 spectrometer at iThemba in South Africa demonstrates that the earlier observation of a state at 11.16 $\mathrm{MeV}$ was almost certainly an experimental artifact and no such state exists [22].

If the Hoyle-state is more extended than the ground-state (as suggested in Fig. 3), and the system behaves in a rotational fashion, then the $2^{+}$state would be lower in energy. Alternatively, the Hoylestate could possess no collective excitations. Studies of the ${ }^{12} \mathrm{C}\left(\alpha, \alpha^{\prime}\right)$ and ${ }^{12} \mathrm{C}\left(\mathrm{p}, \mathrm{p}^{\prime}\right)$ [23] reactions indicate the presence of a $2^{+}$state close to $9.6-9.7 \mathrm{MeV}$ with a width of 0.5 to $1 \mathrm{MeV}$. The state is only weakly populated in these reactions, presumably due to its underlying cluster structure, and is broad. Consequently, its distinction from other broad-states and dominant collective excitations (e.g. the $9.6 \mathrm{MeV}, 3^{-}$state) made its unambiguous identification in these measurements challenging. Further, and perhaps definitive, evidence for such an excitation comes from measurements of the ${ }^{12} \mathrm{C}(\gamma, 3 \alpha)$ reaction performed at the HIGS facility, TUNL [24]. Here a measurable cross section for this process was observed in the same region of 9-10 MeV which cannot be attributed to other known states in this region. Furthermore, the angular distributions of the $\alpha$-particles are consistent with an $L=2$ pattern, demonstrating a dominant $2^{+}$component.

Based on a rather simple description of this state in terms of three $\alpha$-particles with radii given by the experimental charge radius, it is possible to use the $2 \mathrm{MeV}$ separation between the Hoyle-state and the proposed $2^{+}$excitation to draw some conclusions as to the arrangements of the clusters [23]. A linear arrangement of the $3 \alpha$-particles, in which the separation is close to that of the ${ }^{8} \mathrm{Be}$ ground state, 
would give a separation of between the Hoyle-state and the $2^{+}$state of close to $1 \mathrm{MeV}-$ as opposed to the $2 \mathrm{MeV}$ that is observed experimentally. The data would then indicate that rather than a linear arrangement of the three clusters, a more appropriate description would be a loose arrangement of the $\alpha$-particles in something approaching a triangular structure.

A natural extension of the rotational model is that there should also be a collective $4^{+}$state. Using the simple $J(J+1)$ scaling, a $4^{+}$excitation close to $E_{x}\left({ }^{12} \mathrm{C}\right)=14 \mathrm{MeV}$ would be expected. Measurements of the two reactions ${ }^{9} \mathrm{Be}(\alpha, 3 \alpha) n$ and ${ }^{12} \mathrm{C}(\alpha, 3 \alpha){ }^{4} \mathrm{He}$ have been performed [25]. These measurements indicate a candidate state close to $13.3 \mathrm{MeV}$ with a width estimated to be $1.7 \mathrm{MeV}$. It is believed that this is not a contaminant and is observed with similar properties in all spectra. Angular correlation measurements made using the ${ }^{12} \mathrm{C}$ target are not definitive, but indicate a $4^{+}$assignment.

\subsection{Dynamical Excitations of the Ground State}

As indicated in Fig. 3 the triangular structure seen in the AMD calculations for the Hoyle-state is also found in the ground-state. Here the $\alpha$-particles exist in a more compact configuration and at such separations the internal fermionic degrees of freedom become important and the Pauli principle plays a role in perturbing the cluster structure. It is well-known that the ground-state has a significant overlap with shell-model like structures, whereas approaches the NCSM [19] fail to reproduce the properties of the Hoyle-state.

Nevertheless, the triangular symmetry that is observed in the calculations may also be found in the experimental spectroscopic fingerprint. The rotations corresponding to $K^{\pi}=0^{+}$have long been known to correspond to the $0^{+}$ground state, $2^{+} 4.4 \mathrm{MeV}$ and $4^{+} 14.1 \mathrm{MeV}$ excited states. The next collective rotation occurs when each $\alpha$-particle is provided with one unit of angular momentum around the 3 -fold symmetry axis that passes through the centre of the triangle. These three units of angular momenta are linked to $K^{\pi}=3^{-}$. The collective excitations are then $3^{-}, 4^{-}, 5^{-} \ldots$. A precision measurement of the width of the $9.64 \mathrm{MeV}, 3^{-}$, state has recently been published [26] indicating that $\alpha$-clustering may play a non-negligible role in the structure of this state. A collective $4^{-}$excitation of this state has been suggested at $13.35 \mathrm{MeV}[27,28]$, with a $5^{-}$member at $22.4 \mathrm{MeV}$ [29]. The rotational behaviour of the ground-state rotational band and the $K^{\pi}=3^{-}$band (associated with the $9.64 \mathrm{MeV}$ state) are indicative of a $D_{3 h}$ dynamical symmetry reflecting the underlying triangular $3 \alpha$-structure of ${ }^{12} \mathrm{C}$ [29].

\section{The $4 \alpha$ system ${ }^{16} 0$}

The work by Hafstad and Teller [20] indicates the collective properties of the $4 \alpha$ system should be described by the tetrahedral symmetry group; $T_{d}$. Here the properties are those of a spherical top, with equal moments of inertia. If one assumes the separation of the $\alpha$-particles is that which is associated with the ${ }^{8} \mathrm{Be}$ ground state, $\mathcal{I}_{B e}$, then the rotational energies are given by

$$
E_{J}=\hbar^{2} \frac{J(J+1)}{4 \mathcal{I}_{B e}}
$$

The rotation of the tetrahedral structure corresponds to the equivalent rotation of two ${ }^{8} \mathrm{Be}$ nuclei around their symmetry axis and hence the $4 \mathcal{I}_{B e}$ in the denominator. The symmetry then dictates that all values of $J$ are permitted except $J=1,2$ and 5; states with $J=0,4$ and 8 have even parity and $J=3$, 7 and 11 have negative parity. A key feature of this structure would be degenerate $6^{+}$and $6^{-}$states. A similar conclusion can be found in the recent work of Bijker and Iachello [30]. The experimentally observed states at $6.130 \mathrm{MeV}, 3^{-} ; 10.356,4^{+}$and $21.052 \mathrm{MeV}^{+}$have been linked in this latter work to the collective excitations of the tetrahedral structure. These same calculations predicted states at 
$6.132,10.220$ and $21.462 \mathrm{MeV}$ and electromagnetic transition strengths $B(E 3)$ and $B(E 4)$ of 181 and $338 \mathrm{e}^{2} \mathrm{fm}^{2 L}$ compared with experimental values of 205(10) and 378(133) $\mathrm{e}^{2} \mathrm{fm}^{2 L}$. The comparison between experiment and theory is compelling.

An alternative theoretical approach is provided by the Alpha Cluster Model (ACM) calculations of Bauhoff, Shultheis and Shultheis [11]. These calculations identify a number of cluster structures, including a tetrahedral arrangement of the four $\alpha$-particles in the ground-state. In addition, a planar arrangement of $\alpha$-particles is found for the first excited $0^{+}$state. These structures gives rise to rotational bands. The main difference between the ACM and Algebraic Cluster Model (ACM') of Ref. [30] is evident in the assignment of the $10.356 \mathrm{MeV}^{+}$state. The ACM assigns it to the planar rotational structure, whereas as noted above the ACM' links it to the tetrahedral ground-state. What is clear from measurements of the $\alpha$-decay branching ratios for decay to the ${ }^{12} \mathrm{C}$ ground state and first excited states is that the states in the ACM planar band, above the alpha-decay threshold, all have very similar decay properties - they predominantly decay to the ground state [31]. This similar structure conflicts with the tetrahedral interpretation and indicates a collective excitation built around a ${ }^{12} C+\alpha$ cluster structure where the total angular momentum of the state is generated by the orbital motion of the $\alpha$-particle around the ${ }^{12} \mathrm{C}$ core.

To arrive at a better understanding of the cluster symmetries of ${ }^{16} \mathrm{O}$ further electromagnetic transition strengths need to be determined. These include states above the $\alpha$-decay threshold, where small branching ratios $\left(<10^{-5}\right)$ make such studies very challenging.

\section{Summary}

One of the original predictions of the structure of light nuclei [20] suggested the ground state of nuclei such as ${ }^{8} \mathrm{Be},{ }^{12} \mathrm{C}$ and ${ }^{16} \mathrm{O}$ are composed of geometric arrangements of $\alpha$-particles. Though this idea has largely fallen from favour, it is now clear that the two nuclei ${ }^{8} \mathrm{Be}$ and ${ }^{12} \mathrm{C}$ have a spectroscopic finger print that reveals this underlying cluster structure. In these systems the cluster symmetries remain, even if the clusters themselves are not completely intact. The $4 \alpha$ system ${ }^{16} \mathrm{O}$ presents the next challenge to our understanding. Within a model which exploits the dynamical symmetries of the tetrahedral system, $T_{d}$, there appears to be a reasonable basis for the description of the experimental states. However, there remain inconsistencies from the experimental perspective, which mean that there remains experimental challenges to provide data that can definitively constrain the symmetries and structure.

\section{Acknowledgements}

The author would like to acknowledge the contribution from his colleagues at Birmingham who have contributed significantly through the experimental programme that underpins the present contribution.

\section{References}

[1] K. Ikeda, et al., Prog. Theor. Phys. Suppl., Extra Numbers, 464 (1968)

[2] M. Freer and H.O.U. Fynbo, Progress in Particle and Nuclear Physics 78, 1 (2014)

[3] E. Öpik, Proceedings of the Royal Irish Academy A 54, 49 (1951)

[4] E.E. Salpeter, Astrophysical Journal 115, 326 (1952)

[5] F. Hoyle, Astrophysical Journal, Supplement Series 1, 12 (1954)

[6] D.N.F. Dunbar, R.E. Pixley, W.A. Wenzel and W. Whaling, Phys. Rev. 92, 649 (1953) 
[7] C.W. Cook, W.A. Fowler, C.C. Lauritsen and T. Lane, Phys. Rev. 107, 508 (1957)

[8] J.D. Barrow and F.J. Tipler, The Anthropic Cosmological Principle, Cambridge University Press (1986)

[9] B. Carter, Confrontation of Cosmological Theories with Observational Data, Dordecht: Reidel 291 (1974)

[10] H. Kragh, Archive for History of Exact Sciences 64, 721 (2010)

[11] W. Bauhoff, H. Schultheis, and R. Schultheis, Phys. Rev. C 29, 1046 (1984)

[12] J. Okołowicz, W. Nazarewicz and M. Płoszajczak, Fortschritte der Physik 61, 66 (2013)

[13] M. Freer, Nature 487, 309 (2012)

[14] R. Ludwig, Angew. Chem. Int. Ed., 401808 (2001)

[15] F.N. Keutsch and R. J. Saykally, PNAS 98, 10533 (2001)

[16] Y. Kanada En'yo Prog. Theor. Phys. 117, 655 (2007)

[17] T. Marumori, J. Phys. Soc. Jpn. 44, 225 (1978); M. Kamimura, Nucl. Phys. A351, 456 (1981)

[18] E. Uegaki, S. Okabe, Y. Abe and H. Tanaka, Prog. Theor. Phys. 57, 1262 (1977)

[19] P. Navrátil, et al., Phys. Rev. Lett. 84, 5728 (2000)

[20] L. R. Hafstad, E. Teller, Phys. Rev. 54, 681 (1938)

[21] G. M. Reynolds, D. E. Rundquist and R. M. Poichar, Phys. Rev. C 3, 442 (1971)

[22] F.D. Smit, et al., Phys. Rev. C 86, 037301 (2012)

[23] M. Itoh et al.; Nucl. Phys. A738, 268 (2004); Phys. Rev. C 84, 054308 (2011); M. Freer et al.;

Phys. Rev. C 80, 041303(R) (2009); W.R. Zimmerman et al.; Phys. Rev. C 84, 027304(BR) (2011).

[24] W.R. Zimmerman et al.; Phys. Rev. Lett. 110, 152502 (2013).

[25] M. Freer et al.; Phys. Rev. C 83, 034314 (2011).

[26] Tz. Kokalova, M. Freer, Z. Buthelezi, J. Carter, R. W. Fearick, S. V. Förtsch, H. Fujita, R.

Neveling, P. Papka, F. D. Smit, J. A. Swartz, and I. Usman Phys. Rev. C 87, 057307 (2013)

[27] M. Freer et al., Phys. Rev. C 76, 034320 (2007)

[28] O.S. Kirsebom et al., Phys. Rev. C 81, 064313 (2010)

[29] D.J. Marín-Lambarri, R. Bijker, M. Freer, M. Gai, Tz. Kokalova, D.J. Parker, C. Wheldon, Phys.

Rev. Lett. 113, 012502 (2014)

[30] R. Bijker and F. Iachello, Phys. Rev. Lett. 112, 152501 (2014)

[31] C. Wheldon, et al., Phys. Rev. C 83, 064324 (2011) 